\title{
CORRELACIÓN ENTRE CARACTERÍSTICAS FÍSICAS NEONATALES Y MATERNAS CON EL RECUENTO TOTAL DE CÉLULAS NUCLEADAS Y DE CÉLULAS CD34+ POR MICROLITRO EN SANGRE DE CORDÓN UMBILICAL
}

\author{
FELIPE RODRÍGUEZ ORDÓÑEZ ${ }^{1}$, DAVID PÉREZ RIAÑO ${ }^{1}$, ANGÉLICA LEÓN MARTÍN ${ }^{1}$, ALEJANDRA \\ MOSQUERA ZÁRATE', ALEXANDRA ROJAS ÁVILA², CARLOS AUGUSTO FORERO VILLAMIL', \\ XIMENA ROMERO INFANTE", ANGELA RIVEROS AROCHA ${ }^{5}$, LUZ MABEL ÁVILA-PORTILLO'.
}

${ }^{1}$ Médico General, Grupo de Investigación Medicina Materno Fetal y Ginecología El Bosque.Universidad El Bosque. ${ }^{2}$ Epidemióloga general, Magister en Salud Pública. Grupo de Investigación Medicina Materno Fetal y Ginecología El Bosque. Universidad El Bosque.

${ }^{3}$ Gineco - Obstetra, Clínica El Bosque. Magister Bioética. Grupo de Investigación Medicina Materno Fetal y Ginecología El Bosque. Universidad El Bosque.

${ }^{4}$ Gineco - Obstetra, especialista en Medicina Materno Fetal. Líder Grupo de

Investigación Medicina Materno Fetal y Ginecología El Bosque, Universidad El Bosque, Ecodiagnóstico El Bosque. Unidad Medicina Materno Fetal, Clínica El Bosque.

${ }^{5}$ Bacterióloga. Stem Medicina Regenerativa - Banco de Células Madre de Colombia, Bogotá, Colombia. ${ }^{6}$ Magister en ciencias biomédicas. cPhD Ciencias farmacéuticas. Stem Medicina Regenerativa - Banco de Células Madre de Colombia, Bogotá, Colombia.

Correspondencia:Luz Mabel Ávila Portillo mabelavila_us@yahoo.com

Recibido: Mayo 4 de 2015 Aceptado: Julio 31 de 2015

\begin{abstract}
Resumen
Introducción: La sangre de cordón umbilical (SCU) ha sido reconocida como una fuente de células madre hematopoyéticas. Múltiples estudios han sido realizados con el propósito de determinar variables maternas y neonatales que afecten el volumen, el recuento total de células nucleadas y de células CD34+. El presente estudio correlaciona variables maternas y neonatales con el recuento total de células nucleadas y de CD34+ medidas en $\mu \mathrm{L}$ (microlitro).

Materiales y Métodos:Estudio correlacional en el que se analizaron 50 muestras de sangre de cordón umbilical de gestantes atendidas en dos IPS, una de Bogotá y otra de Ubaté, (Cundinamarca), durante un período de 7 meses del año 2013. La selección de estas muestras fue basada en un muestreo no probabilístico. Se calculó el coeficiente de correlación de Pearson con su respectiva significancia estadística entre las variables cuantitativas de la madre y del neonato, y el volumen, el recuento de células CD34+ y el recuento total de células nucleadas por $\mu \mathrm{L}$.

Resultados:Se encontró correlación positiva entre la longitud del cordón y el recuento total de células nucleadas. Así mismo entre el volumen inicial de la muestra y el recuento total de células nucleadas y el recuento de células $\mathrm{CD} 34+$ en $\mu \mathrm{L}$ y en $\mathrm{mL}$ (mililitro).

Discusión:Múltiples estudios se han desarrollado entorno a la búsqueda de variables maternas y neonatales que afectan la calidad de la muestra. En Colombia no se habían descrito las correlaciones encontradas en el presente estudio. Es por ello, que el desarrollo de nuevos estudios con diseños analíticos será enriquecedor. Palabras claves: Células madre, antígeno $\mathrm{CD}_{34+}$, trasplante de células madre de sangre de cordón umbilical (SCU), neonatal, materno, factores de riesgo.
\end{abstract}




\title{
CORRELATION BETWEEN NEONATAL AND MATERNAL PHYSICAL CHARACTERISTICS WITH TOTAL NUCLEATED CELL COUNT AND CELL COUNT PER MICROLITER CD34+ IN CORD BLOOD
}

\begin{abstract}
Background: The cord blood has been recognized as a source of hematopoietic stem. Multiple studies have been conducted in order to determine maternal and neonatal variables that affect the volume, total nucleated cell count and CD34+ cells. This study correlated maternal and neonatal variables with the total nucleated cell count and CD34+ cells measured by $\mu \mathrm{L}$.

Materials and methods: Correlational study in which 50 samples of umbilical cord blood were analyzed of pregnant women at two health institutions, one at Bogotá and the other one at Ubaté, Cundinamarca, for a period of seven months of 2013. The selection of these samples was based on a non-probability sample. The Pearson correlation coefficient was calculated with their respective statistical significance between quantitative variables of mother and newborn, and the volume, the count of CD34+ cells and the total nucleated cell count measured by $\mu \mathrm{L}$.

Results: Positive correlation between the length of the umbilical cord and the total nucleated cell count was found. Similarly, correlation between total nucleated cell count with the initial volume and count of CD34+ cells in $\mu \mathrm{L}$ and $\mathrm{mL}$ was found.

Discussion: Several studies have been conducted around to look maternal and neonatal variables that affect the sample quality, however, the Colombian literature has not described the correlation found in this study, so it requires the development of new research with designs of analytical studies to establish associations between the variables described.

Key words: Stem cells, antigens CD34+, umbilical cord blood cell transplantation, neonatal, maternal, risk factors.

\section{CORRELAÇÃO ENTRE CARACTERÍSTICAS FÍSICAS NEONATAIS E MATERNAS COM A CONTAGEM TOTAL DE CÉLULAS NUCLEADAS E CÉLULAS CD34 + POR MICROLITRO NO SANGUE DO CORDÃO UMBILICAL}

\section{Resumo}

Introdução: O sangue do cordão umbilical (SCU) tem sido reconhecido como uma fonte de células-tronco hematopoiéticas. Vários estudos têm sido realizados com a finalidade de determinar as variáveis maternas e neonatais que afetam o volume e a contagem total de células nucleadas e células $\mathrm{CD} 34+$. O presente estudo correlaciona as variáveis maternas e neonatais com a contagem total das células nucleadas e células CD34+ medidas em $\mu \mathrm{L}$ (microlitro).

Materiais e métodos: Estudo correlacional no que 50 amostras de sangue do cordão umbilical de mulheres grávidas atendidas em dois IPs foram analisadas, uma em Bogota e outra em Ubaté (Cundinamarca), durante um período de 7 meses do ano 2013. A seleção destas amostras foi baseada em uma amostragem não probabilística. O coeficiente de correlação de Pearson foi calculado com a respectiva significância estatística entre variáveis quantitativas da mãe e do recém-nascido, assim como o volume, a contagem de células CD34 + e contagem total de células nucleadas por $\mu \mathrm{L}$.

Resultados: Correlação positiva foi encontrada entre o comprimento do cordão e a contagem total de células nucleadas. Também entre o volume da amostra inicial e contagem total de células nucleadas e contagem de células $\mathrm{CD} 34+$ em $\mu \mathrm{L}$ e em $\mathrm{mL}$ (mililitro).

Discussão: Vários estudos têm sido desenvolvidos em torno da busca de variáveis maternas e neonatais que afetam a qualidade da amostra. Na Colômbia não tinham sido descritas as correlações encontradas no presente estudo. É por esta razão que será gratificante o desenvolvimento de novos estudos com desenhos analíticos.

Palavras-chave: Células-tronco, antígeno $\mathrm{CD}^{2}{ }^{+}$, transplante de células-tronco do sangue do cordão umbilical (SCU), neonatal, materno, fatores de risco. 


\section{Introducción}

Desde la década de los ochentas se ha reconocido a la sangre de cordón umbilical (SCU) como una fuente rica en células madre hematopoyéticas, siendo estas de gran utilidad en el manejo y tratamiento de desórdenes hematológicos malignos y no malignos, desórdenes músculo esqueléticos, endocrinos, enfermedades cardiovasculares, inmunológicas, trastornos neurológicos, entre otros, razón por lo cual se considera hoy en día la necesidad de criopreservarla (1-8). Aunque se observan muchas ventajas en comparación con las muestras obtenidas de médula ósea, la mayor desventaja es el bajo recuento de células progenitoras hematopoyéticas unido al volumen de la muestra obtenido (7-10).

Hasta el momento, se ha tenido en cuenta el volumen y el recuento total de células nucleadas como variables principales para la criopreservación de las muestras $(6,11-14)$. El recuento de células $\mathrm{CD} 34+$ y el recuento total de células nucleadas han sido utilizados como parámetros para evaluar la calidad de la muestra (9), siendo el recuento de las células CD34+ el parámetro para evaluar el potencial hematopoyético de la muestra y el recuento total de células nucleadas el indicador de resultados de trasplantes y por ende de mortalidad (7,15-22). Un tratamiento efectivo requiere una muestra de SCU con aproximadamente 2 2,5 X 107 células nucleadas por $\mathrm{kg}$ de peso $(8,15,23-24)$ y 2 X 105 - 106 células CD34+ por kg de peso $(6,19)$. Sin embargo, solamente el $25 \%$ de las muestras recolectadas con volúmenes mayores a $80 \mathrm{ml}$ contienen un adecuado recuento celular para pacientes con pesos entre 50 y $70 \mathrm{~kg}$ (15). Lo que sugiere que otras variables, a parte del volumen de la muestra, influyen en el recuento total de células nucleadas y de células $\mathrm{CD} 34+$.

Desde el primer trasplante realizado de células madre de sangre de cordón umbilical $(15,25)$, múltiples estudios se han desarrollado en pro de optimizar el proceso de selección del donante con el fin de encontrar correlación entre variables maternas y neonatales con la calidad de la muestra $(8,9,22,25)$, utilizando como unidad de medida los mililitros, sin embargo, no hay ningún tipo de información en la que se estudien estas variables en $\mu \mathrm{L}$ (microlitro).

\section{Materiales y Métodos}

\section{Diseño del estudio}

Estudio correlacional que determina los factores fisiológicos del neonato y patológicos de base de la madre que se correlacionan con la calidad de la muestra de SCU.

\section{Población y tamaño de muestra}

50 mujeres gestantes, que asistieron a la Clínica El Bosque y al Hospital El Salvador de Ubaté durante un período de 7 meses, comprendido entre mayo y noviembre del año 2013.

\section{Variables}

Se tomaron como variables los datos clínicos maternos y neonatales, tales como: edad de la madre, edad gestacional, paridad, patologías maternas, vía del parto; sexo, peso y talla neonatal; características físicas del cordón umbilical como diámetro y longitud, y peso de la placenta.

\section{Procedimiento}

Con previa aceptación voluntaria de las gestantes y familiares mediante la firma del consentimiento informado, aprobado por el Comité Institucional de Ética de la Universidad El Bosque, se solicitó la historia clínica de la paciente a la institución con el fin de obtener datos maternos, obstétricos y fetales. En el momento del parto y después de la obtención de la muestra de SCU, los investigadores tomaron los datos del neonato tales como; talla, peso, diámetro y longitud de cordón umbilical y peso de la placenta.

Posteriormente, se trasladó la muestra al banco de células madre en una nevera de icopor a una temperatura de -4 grados centígrados $\left(-4^{\circ} \mathrm{C}\right)$ en un tiempo no mayor de 36 horas, en donde se procesó determinando el volumen y el recuento leucocitario a través del hemocontador automatizado BC 2800 (Mindray, High tech industrialm Park, Nahnshan, China).

El recuento de células $\mathrm{CD} 34+$ por citometría de flujo se realizó en el citómetro FACSCantoll (Becton Dickinson USA), con el módulo de aplicación clínica, basados en el protocolo ISAGHE (Figura 1), en un tiempo no mayor a 36 horas. La configuración del equipo previa al montaje de las muestras, se realizó con BD FACS 7-colorbSetup Beads, seguido por la optimización (spillover), al ser satisfactorios los controles se procedió al montaje de las muestras. Siguiendo las instrucciones suministradas del BD ${ }^{\mathrm{TM}}$ Stem Cell Enumeration Kit se prepararon las muestras, agregando $20 \mu \mathrm{l}$ de CD45 FITC/CD34 PE, $20 \mu \mathrm{l}$ de 7aminoactinomycin $\mathrm{D}(7-\mathrm{AAD})$ y $100 \mu \mathrm{L}$ de muestra, mezclado e incubando durante 20 minutos en oscuridad. Al finalizar el tiempo de incubación se agregó $2 \mathrm{ml}$ de solución de lisis (Cloruro de amonio - $\mathrm{NH} 4 \mathrm{Cl}$ ), vortex e incubación por 10 minutos.

La información obtenida tanto de la madre como del recién 
BD Stem Cell + 7AAD
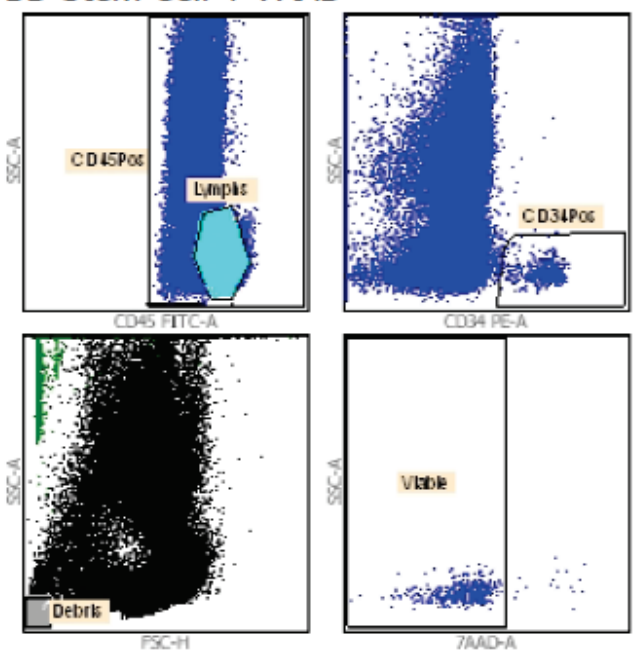

MUESTRA \# 36036.001.fCs

CD34+ Viable Abs Cnt (cells/ul)

CD45+ Viable Abs Cnt (cells/ul)

CD34+ Viable Events

CD45+ Viable Events

Bead Events

Viable CD34+ \% Viable CD45+

CD34 Viability (\%)

Viable CD34+ CV (\%)
Eventos totales: 110779
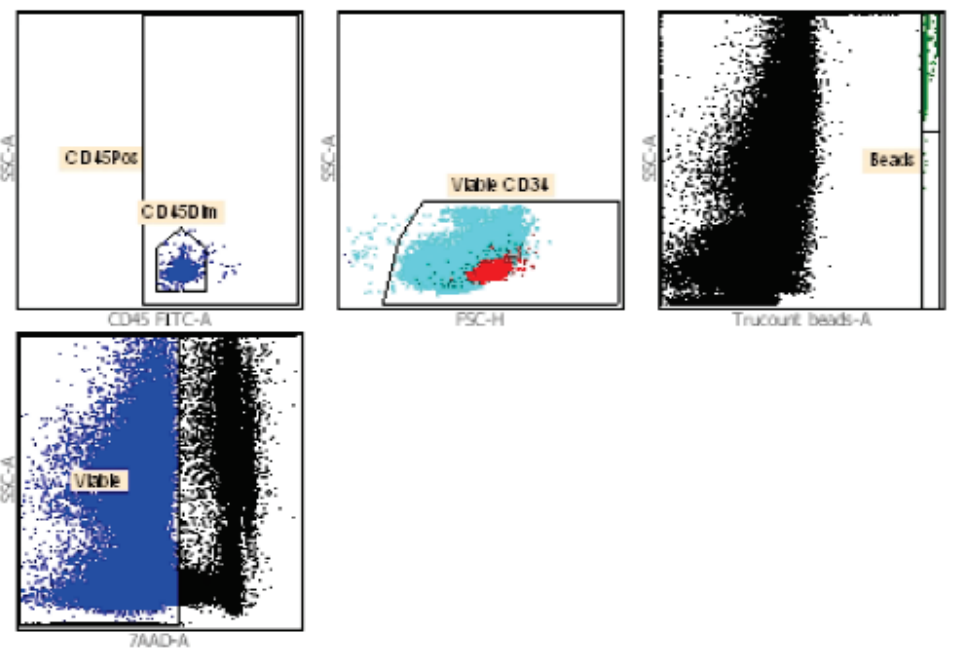

ID de lote: 4020970
61.13

5562.88

830

75525

7311

1.10

97.88

3.47

Figura 1. Gráficos de puntos obtenidos en el módulo de aplicación BD SCE de BDFACSCanto software clínico.

nacido fue documentada en el formato de recolección de datos, y posteriormente, procesada en el programa estadístico Excel versión 2007, siguiendo con la importación de los datos al programa estadístico informático SPSS versión 20.0 con licencia para el Banco de Células Madre de Colombia.

Durante la ejecución de este proyecto se tuvieron en cuenta las normas científicas, técnicas, administrativas y éticas para la investigación en salud establecidas en la resolución No. 8430 de 1993 del Ministerio de Salud, la cual cataloga este estudio como "Investigación de riesgo mínimo", contemplando el título I, capítulos I y título II, capítulo 1, artículo 11, para la investigación en humanos.

\section{Análisis de los datos}

Se realizó un análisis descriptivo de la información, a través del cálculo de medidas de tendencia central para las variables cuantitativas, y porcentajes y frecuencias para las variables cualitativas. Posteriormente, se calculó el coeficiente de Pearson con su respectivo nivel de significancia para las variables cuantitativas de la madre y el neonato frente a los marcadores de calidad; a saber, el recuento total de células $\mathrm{CD} 34+$, el recuento total de células nucleadas y el volumen inicial de la muestra, todas medidas en microlitros. Para determinar diferencia de medias del recuento total de células CD34+ y el recuento total de células nucleadas según sexo y paridad, se calculó la prueba estadística t de student con su respectivo nivel de significancia.

\section{Resultados}

Un total de 50 muestras fueron analizadas. La edad promedio de las gestantes fue de $24,4 \pm 5,7$ años y la edad gestacional promedio de $38,7 \pm 5,1$ semanas. El peso promedio del neonato de $3118,6 \pm 371$ gr y la estatura promedio $49,9 \pm 2,3 \mathrm{~cm}$. El peso promedio de la placenta de $562 \pm 108$ gr, la longitud del cordón umbilical de 52,3 \pm $13 \mathrm{~cm}$ y el diámetro del cordón umbilical de $1,2 \pm 0,3 \mathrm{~cm}$ (Tabla 1). 
Tabla 1. Características cuantitativas fisiológicas del neonato y patológicas de las gestantes estudiadas

\begin{tabular}{|c|c|c|c|c|c|c|}
\hline Variable & Parámetro de medición & Media & Mediana & $\begin{array}{l}\text { Desviación } \\
\text { típica }\end{array}$ & Valor mínimo & Valor máximo \\
\hline Peso placenta & gramos & 562 & 550,0 & 108,9 & 310 & 850 \\
\hline Diámetro cordón & centímetros & 1,22 & 1,2 & 0,3 & 0,7 & 2 \\
\hline Talla recién nacido & centímetros & 49,92 & 50,0 & 2,3 & 43 & 57 \\
\hline
\end{tabular}

Según el tipo de atención del parto, el 71\% fue vía vaginal, el $27 \%$ cesárea y un $2 \%$ parto vaginal instrumentado. Según el sexo de los neonatos, el $58 \%$ fueron masculinos y el $42 \%$ femeninos.

El volumen promedio de la muestra inicial fue de 41,4 \pm 23 $\mathrm{ml}$ y el recuento total promedio de células nucleadas de $564.359 .432,7 \pm 343.748 .601,9$. El recuento promedio de células CD34+ fue de 1.890.502 $\pm 1.525 .099,2$. De igual forma, el recuento total promedio de células nucleadas y CD34+ por $\mu \mathrm{L}$ (microlitro), fue de 7.138,0 $\pm 2.659,6$ y $22,47 \pm 13,6$ respectivamente. (Tabla 2 )

Tabla 2. Características físicas y celulares de las muestras analizadas

\begin{tabular}{|c|c|c|c|c|c|c|}
\hline Variable & Parámetro de medición & Media & Mediana & $\begin{array}{l}\text { Desviación } \\
\text { típica }\end{array}$ & Valor mínimo & Valor máximo \\
\hline Volumen inicial & millilitro & 41,4630 & 35,0 & 23,2 & 9,02 & 107,23 \\
\hline $\begin{array}{l}\text { Recuento células } \\
\text { nucleadas }\end{array}$ & células/microlitro & 7138 & 7250,0 & 2659,6 & 1800 & 12600 \\
\hline $\begin{array}{l}\text { Recuento celular } \\
\text { total }\end{array}$ & $\begin{array}{l}\text { células/microlitro } \\
\text { de la muestra. }\end{array}$ & 564359432,74 & 472815197,0 & 343748601,9 & 105658537 & 1582197749 \\
\hline $\begin{array}{l}\text { Recuento celular } \\
\text { CD34+ }\end{array}$ & células/microlitro & 22,47 & 20,3 & 13,7 & 2,09 & 56,3 \\
\hline $\begin{array}{l}\text { Recuento celular } \\
\text { total CD } 34+\end{array}$ & $\begin{array}{l}\text { células/microlitro } \\
\text { de la muestra. }\end{array}$ & 1890502,58 & 1470174,5 & 1525099,3 & 999,37 & 5861326 \\
\hline
\end{tabular}

Se encontró correlación positiva, moderada, entre la longitud del cordón y el recuento total de células nucleadas por $\mu \mathrm{L}$ (microlitro) $\mathrm{r}=0,363(\mathrm{p}<0,05)$. Así mismo, se halló una correlación positiva fuerte, entre el volumen inicial de la muestra y los recuentos totales de células nucleadas $r$ : 0,82 $(p<0,05)$; recuento total de células nucleadas en mililitro $(\mathrm{mL})$ y el recuento de células $\mathrm{CD} 34+$ en microlitro $(\mu \mathrm{L})$ y mililitro $(\mathrm{mL})$ respectivamente, $r$ : $0,71(p=0,015)$

Sin embargo, no se encontró correlación entre el recuento total de células nucleadas en microlitro con el peso de la placenta $(p=0,520)$, el diámetro del cordón $(p=0,937)$, la talla del bebé $(p=0,441)$, el peso del bebé $(p=745)$, la edad gestacional $(p=0,612)$, el sexo $(p=0,343)$, la paridad $(p=0,704)$, ni con la edad materna $(p=0,119)$. Tampoco, se encontró correlación entre las variables maternas y neonatales con el recuento de células CD34+ por $\mu \mathrm{L}$ (microlitro) tales como: edad materna $(p=0,482)$, edad gestacional $(p=0,786)$, peso de la placenta $(p=0,558)$, longitud $(p=0,114)$ y diámetro del cordón umbilical $(p=0,854)$, talla del bebé $(p=896)$, peso del bebé $(p=488)$, sexo $(0,658)$, ni con la paridad $(0,777)$. (Tabla 3$)$ 
Tabla 3. Nivel de significancia de variables cuantitativas maternas y neonatales. Los resultados de la gráfica se obtuvieron a través del cálculo de coeficiente de Pearson con su respectivo nivel de significancia para las variables cuantitativas y para las variables cualitativas se utilizó la prueba estadística tde student de igual forma con su respectivo nivel de significancia.

\begin{tabular}{lcc|} 
Variable & $\begin{array}{c}\text { Recuento } \\
\text { células nucleadas }\end{array}$ & $\begin{array}{c}\text { Recuento } \\
\text { celular CD34+ }\end{array}$ \\
\hline Edad materna &, 119 &, 482 \\
\hline Peso de la placenta &, 520 &, 558 \\
\hline Longitud del cordón &, 010 &, 114 \\
\hline Diámetro del cordón &, 937 &, 854 \\
\hline Edad gestacional &, 612 &, 786 \\
Talla bebe &, 441 &, 896 \\
\hline Peso bebé &, 745 &, 488 \\
\hline
\end{tabular}

De las muestras tomadas solamente se detectaron 4 gestantes con infección de vías urinarias (8\%) y una gestante con diabetes mellitus tipo 2 (2\%). Otro tipo de patologías y situaciones maternas, tales como, asma crónica, epilepsia primaria, incompatibilidad $\mathrm{RH}$, hipertensión arterial crónica, ruptura prematura de membranas, vaginosis bacteriana y parto prolongado fueron reportadas. Dado la baja presentación de patologías maternas en la muestra, no fue posible realizar la prueba estadística para encontrar diferencias entre ellas y el recuento total de células nucleadas y de células CD34+ en $\mu \mathrm{L}$ (microlitro).

\section{Discusión}

En el presente estudio se encontró correlación entre el volumen inicial de la muestra, con el recuento total de células nucleadas y el recuento de células CD34+, encontrando que a mayor volumen mayor recuento de células CD34+ $(p=0,015)$ y recuento total de células nucleadas $(p=0,002)$, hallazgos ya descritos en la literatura(9). Por otro lado, se encontró, que de las variables neonatales, la longitud del cordón fue la única que se correlacionó con el recuento total de células nucleadas y no hubo ninguna otra correlación entre las demás variables maternas o neonatales analizadas como vía del parto, peso materno, edad gestacional, peso de placenta, peso del bebé datos que contrastan en cuanto a que el peso del neonato al momento del nacimiento es el principal factor neonatal que afecta el Numero de CD34+ y Recuento de células nucleadas (710,22,26-31). Llama la atención el hecho que se hubiera encontrado correlación solamente entre la longitud del cordón umbilical y el recuento total de células nucleadas y no también con el recuento de células CD34+, teniendo en cuenta, y según los resultados del presente estudio, la

\begin{tabular}{lcc} 
Variable & $\begin{array}{c}\text { Recuento } \\
\text { células nucleadas }\end{array}$ & $\begin{array}{c}\text { Recuento } \\
\text { celular CD34+ }\end{array}$ \\
Sexo & 0,343 & 0,658 \\
Paridad & 0,704 & 0,777 \\
Volumen &, 002 &, 015 \\
Recuento células nucleadas $(\mu \mathrm{l})$ & - &, 000 \\
\hline $\begin{array}{l}\text { Recuento celular total }(\mathrm{ml}) \\
\text { Recuento celular CD34+ }(\mu \mathrm{ll})\end{array}$ &, 000 &, 000 \\
\hline Recuento celular total $\mathrm{CD} 4+(\mathrm{ml})$ &, 000 & - \\
\hline
\end{tabular}

existencia de correlación entre estos dos marcadores de calidad, inquietudes que valen la pena tener en cuenta para próximas investigaciones.

Todos estos estudios tienen como objetivo buscar factores que afecten la calidad de la muestra, y permitan a los bancos de sangre de cordón umbilical ser más eficientes en la selección de donantes adecuados, y así, mejorar la oportunidad en la utilización de unidades de sangre de cordón umbilical para lograr resultados clínicos satisfactorios en aquellos pacientes a los que se les realice trasplante con células madre de SCU.

Las limitaciones del presente estudio comprenden el pequeño número de pacientes incluidos. A pesar de no ser una muestra grande de pacientes, consideramos que nuestros hallazgos aportan evidencia adicional relacionada con la necesidad de tomar muestras de gran calidad y volumen que conlleven a recuentos altos de células madre hematopoyéticas, sin embargo, consideramos que es necesario ampliar el número de muestras para confirmar estos hallazgos en maternas colombianas. En la literatura colombiana no se ha descrito la correlación encontrada en este estudio ni otro tipo de resultados respecto al objetivo de este estudio, por lo que se amerita el desarrollo de nuevas investigaciones con diseños de estudios analíticos que permitan establecer asociaciones entre las variables descritas.

En conclusión, los hallazgos del presente estudio confirman la correlación entre el volumen inicial de la muestra, con el recuento total de células nucleadas y la correlación entre la longitud del cordón y el recuento total de células nucleadas en $\mu \mathrm{L}$ (microlitro). 


\section{Conflictos de Interés}

No se reportó ningún tipo de conflicto de interés por los investigadores.

\section{Financiación}

\section{Universidad El Bosque y Stem Medicina Regenerativa}

\section{Referencias}

1. Revencu T, Trifan V, Nacu L, Gutium T, Globa L, Motoc AG, et al. Collection, isolation and characterization of the stem cells of umbilical cord blood. Rom J Morphol Embryol. 2013;54(2):291-7.

2. Jager M, Degistirici O, Knipper A, Fischer J, Sager M, Krauspe R. Bone healing and migration of cord blood-derived stem cells into a critical size femoral defect after xenotransplantation. J Bone Miner Res. 2007;22(8):1224-33.

3. Takahashi S, Ooi J, Tomonari A, Konuma T, Tsukada N, OiwaMonna $\mathrm{M}$, et al. Comparative single-institute analysis of cord blood transplantation from unrelated donors with bone marrow or peripheral blood stem-cell transplants from related donors in adult patients with hematologic malignancies after myeloablative conditioning regimen. Blood. 2007;109(3):1322-30

4. Malgieri A, Kantzari E, Patrizi MP, Gambardella S. Bone marrow and umbilical cord blood human mesenchymal stem cells: state of the art. Int J Clin Expo Med. 2010;3(4):248-69.

5. Grewal SS, Barker JN, Davies SM, Wagner JE. Unrelated donor hematopoietic cell transplantation: marrow or umbilical cord blood? Blood. 2003;101(11):4233-44

6. Jaime-Perez JC, Monreal-Robles R, Rodriguez-Romo LN, Mancias-Guerra C, Herrera-Garza JL, Gomez-Almaguer D. Evaluation of volume and total nucleated cell count as cord blood selection parameters: a receiver operating characteristic curve modeling approach. Am J Clin Pathol. 2011;136(5):721-6.

7. Al-Sweedan SA, Musalam L, Obeidat B. Factors predicting the hematopoietic stem cells content of the umbilical cord blood. Transfus Apher Sci. 2013;48(2):247-52.

8. Solves Alcaina P, Perales Marin A, Mirabet Lis V, Brik Spinelli M, Soler Garcia MA, Roig Oltra R. [Donors selection and retrieval of units in an umbilical cord blood bank]. Med Clin (Barc). 2007;129(15):561-5.

9. Chandra T, Afreen S, Kumar A, Singh U. Correlation of umbilical cord blood volume with CD34+ cells concentration International Journal of Blood Transfusion and Immunohematology 2011;1:11-15.

10. Mancinelli F, Tamburini A, Spagnoli A, Malerba C, Suppo G, Lasorella R, et al. Optimizing umbilical cord blood collection: impact of obstetric factors versus quality of cord blood units. Transplant Proc. 2006;38(4):1174-6.

11. Solves P, Carbonell-Uberos F, Mirabet V, Roig R. CD34+ cell content for selecting umbilical cord blood units for cryopreservation. Transfusion. 2007;47(3):552-3.

12. Van Haute I, Lootens N, De Buck K, Verdegen L, Vander Steene $\mathrm{V}$, Desmet $\mathrm{S}$, et al. Selecting cord blood units for storage by CD34+ cell counts. Transfusion. 2005;45(3):4557.

13. Wu JY, Liao C, Xu ZP, Chen JS, Gu SL, Huang YN, et al Banking and transplantation of umbilical cord blood in Guangzhou, China. Cytotherapy. 2006;8(5):488-97.

14. Novelo-Garza B, Limon-Flores A, Guerra-Marquez A, LunaBautista F, Juan-Shum L, Montero I, et al. Establishing a cord blood banking and transplantation program in Mexico: a single institution experience. Transfusion. 2008;48(2):228-36.

Gluckman E. Hematopoietic stem-cell transplants using umbilical-cord blood. NEngl J Med. 2001;344(24):1860-1.

Kurtzberg J, Laughlin M, Graham ML, Smith C, Olson JF, Halperin EC, et al. Placental blood as a source of hematopoietic stem cells for transplantation into unrelated recipients. NEngl J Med. 1996;335(3):157-66.

17. Locatelli F, Rocha V, Chastang C, Arcese W, Michel G, Abecasis M, et al. Factors associated with outcome after cord blood transplantation in children with acute leukemia. Eurocord-Cord Blood Transplant Group. Blood. 1999;93(11):3662-71.

18. Laughlin MJ, Barker J, Bambach B, Koc ON, Rizzieri DA Wagner JE, et al. Hematopoietic engraftment and survival in adult recipients of umbilical-cord blood from unrelated donors. NEngl J Med. 2001;344(24):1815-22.

19. Migliaccio AR, Adamson JW, Stevens CE, Dobrila NL, Carrier CM, Rubinstein P. Cell dose and speed of engraftment in placental/umbilical cord blood transplantation: graft progenitor cell content is a better predictor than nucleated cel quantity. Blood. 2000;96(8):2717-22.

20. Shahrokhi S, Menaa F, Alimoghaddam K, McGuckin C, Ebtekar M. Insights and hopes in umbilical cord blood stem cel transplantations. J Biomed Biotechnol. 2012;2012:572821

21. Aroviita P, Teramo K, Westman P, Hiilesmaa V, Kekomaki R. Associations among nucleated cell, CD34+ cell and colonyforming cell contents in cord blood units obtained through a standardized banking process. Vox sang. 2003;84(3):219-27.

22. Wahid FS, Nasaruddin MZ, Idris MR, Tusimin M, Tumian NR, Mahdy ZA. Effects of preeclampsia on the yield of hematopoietic stem cells obtained from umbilical cord blood at delivery. J Obstet Gynaecol Res. 2012;38(3):490-7.

23. Oran B, Shpall E. Umbilical cord blood transplantation: a maturing technology. Hematology Am Soc Hematol Educ Program. 2012;2012:215-22.

24. Rocha V, Gluckman E, Eurocord-Netcord r, European B, Marrow Transplant g. Improving outcomes of cord blood transplantation: HLA matching, cell dose and other graft- and transplantation-related factors. Br J Haematol. 2009;147(2):262-74.

25. Coldwell KE, Lee SJ, Kean J, Khoo CP, Tsaknakis G, Smythe $\mathrm{J}$, et al. Effects of obstetric factors and storage temperatures on the yield of endothelial colony forming cells from umbilical cord blood. Angiogenesis. 2011;14(3):381-92

26. Hussein AA, Bawadi RM, Tahtamouni LH, Frangoul H, Elkarmi AZ. Feasibility of collecting umbilical cord blood in jordan and the effect of maternal and neonatal factors on hematopoietic stem cell content. Mediterr J Hematol Infect Dis. 2014;6(1):e2014019.

27. Jan $\mathrm{RH}$, Wen $\mathrm{SH}$, Shyr MH, Chiang BL. Impact of maternal and neonatal factors on CD34+ cell count, total nucleated cells, and volume of cord blood. Pediatr Transplant. 2008;12(8):868-73.

28. Solves P, Perales A, Moraga R, Saucedo E, Soler MA, Monleon J. Maternal, neonatal and collection factors influencing the haematopoietic content of cord blood units. Acta Haematol. 2005;113(4):241-6.

29. Jones J, Stevens CE, Rubinstein P, Robertazzi RR, Kerr A, Cabbad MF. Obstetric predictors of placental/umbilical cord blood volume for transplantation. Am J Obstet Gynaecol. 2003;188(2):503-9.

30. Manegold-Brauer G, Borner B, Bucher G, Hoesli H, Passweg $\mathrm{J}$, Girsberger S, et al. A penatal prediction model for total nuecleated cell count increases the efficacy of umbilical cord blood banking. Transfusion. 2014:54(11):2946-2952.

31. Chandra T, Afreen S, Kumar A, Singh U, Gupta A. Does umbilical cord blood-derived CD34+ cell concentration depend on the weight and sex of a full-term infant. J Pediatr Hematol Oncol. 2012;34(3):184-7 